\title{
A project to improve process of quality assurance system at the laboratory of District General Hospital Kalutara using adapted Lang's framework model for change
}

\author{
Dr. D.H.N.S. Chandradasa* \\ Registrar in Medical Administration, Ministry of Health .Sri Lanka \\ Dr. K.C. Shanthi Dalpadadu** \\ Consultant Community Physician, Senior fellow, Institute for Health Policy, Sri Lanka \\ DOI: 10.29322/IJSRP.10.10.2020.p10610 \\ http://dx.doi.org/10.29322/IJSRP.10.10.2020.p10610
}

\section{Abstract- Introduction}

Reliability, accuracy and timeliness of laboratory test results are

very important in laboratory Quality Assurance (QA). Accurate test results helps to prevent unnecessary treatment, treatment complications, delays in correct diagnosis and unnecessary diagnostic testing. Laboratory QA cycle consists of preanalytical, analytical and the post analytical stages which affect the accuracy of test results.

District General Hospital Kalutara (DGHK) is one of the largest health care institutions in Kalutara district. This Hospital serves not only people of Kalutara district but also those from the other districts such as Rathnapura and Galle. This project aimed to improve the process QA at the laboratory of DGHK using adapted Lang's framework model for change.

\section{$\underline{\text { Methodology and Results }}$}

The pre interventional phase was to assess the process of QA at DGHK and to identify gaps in quality assurance in the laboratory processes and practices of medical laboratory technologists and nursing officers. Both qualitative and quantitative techniques were conducted during this phase for data collection.

It was observed that there were issues in pre-analytical, analytical and the post analytical stages of laboratory cycle. Specimen collection, specimen quality and the specimen transport in pre-analytical stage, equipment errors and other related errors in the procedures in analytical stage and results reporting, record keeping, and distribution of reports in post analytical stage were selected as major problems. Therefore a package of interventions was undertaken to address above issues and was developed using adapted Lang's framework model for change at DGHK to improve QA system. All the medical wards and short staying unit at Emergency Treatment Unit were prioritized and selected for interventions as majority of samples were sent by them.

QA committee was established and a nonconformity handling form was developed and implemented. Further, sample collection manual was prepared. Sample collection counter and facilitator service was introduced and an internal circular was issued for providing guidance for the new interventions. Initial steps were 
undertaken to establish a Laboratory Information Management System.

Post interventional evaluation was done using the same techniques that were used in the pre intervention phase to assess the effectiveness of the interventions. Desk review of sample register book and other relevant documents revealed that there was a significant reduction in turnaround time after implementing the interventions

\section{Conclusions}

Interventions were effective in improving total QA Process with reducing laboratory errors in all three stages of cycle.

Sample collection manual had helped in reduction of errors in relation to specimen collection, quality, and the transportation. Sample collection counter, the record keeping mechanisms and report delivery systems were effective in improving post analytical errors. There was a significant reduction in turnaround time after the interventions.

It is recommended to implement this project in other units of the DGHK and in other institutions of the country for quality assurance of laboratory process.

Index Terms- Quality Assurance, District General Hospital Kalutara, pre-analytical stage, analytical stage and the post analytical stage

\section{INTRODUCTION}

\section{Background information}

Ministry of Health is committed to improve and strengthen government health sector. In this context laboratory services plays a vital role in the patient care services and this sector need to be improved by strengthening Quality Assurance (QA) of the services they provide.

QA is a management method that is defined as "all those planned and systematic actions needed to be provide adequate confidence that a product service or result will satisfy given requirement for quality and be fit for use". A quality assurance programme is defined as "the sum total of the activities aimed at achieving that required standards (ISO 1994). The WHO (World Health Organisation) definition of QA is a total process whereby the quality of lab reports can be guaranteed.

Activities of QA may be varied and numerous, including research, clinical audit, setting monitoring of standards, evidence-based practice, continuing professional development, lifelong learning, and infection control, health and safety.

There are varieties of conceptual models that have been published as models for evaluation. One such model Lang's framework for change (Annexure 1) has been used successfully as a model for QA programme (QAP).

\section{Figure 1: QA cycle adapted from Lang's cycle}

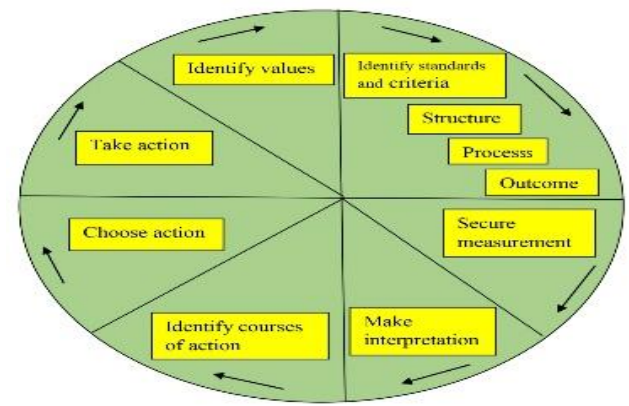

QA cycle is used to assure the quality of care. It consists of identifying values, setting objectives, describing patient care 
in measurable terms, securing measurement, evaluating the results and act completing the cycle.

\section{$\underline{\text { Objectives }}$}

\section{- General objective}

To improve the process of Quality Assurance System (QAS) at the laboratory of DGHK using adapted Lang's framework model for change.

\section{- Specific objectives}

1. To evaluate the existing process and practices to identify gaps in the QAS at the laboratory of DGHK.

2. To develop appropriate interventions to identified gaps using adapted Lang's framework model for change at the laboratory of DGHK.

3. To implement the interventions to improve the QAS in the laboratory process at the laboratory of DGHK.

4. To assess the effectiveness of the process after implementing the interventions to improve the QAS at the laboratory of DGHK.

\section{Methodology}

This was a hospital based interventional study.

\section{Setting}

Project was implemented at DGHK. This is the largest government hospital in Kalutara district. It serves as the final referral center in the district .Haematology and biochemistry sections of the laboratory that carries out Full Blood Count (FBC), Erythrocyte Sedimentation Rate (ESR), Prothrombin
Time/International Normalized Ratio (PT/INR), Lipid Profile, Liver Function Test, Serum Electrolytes were selected for this project. Male and Female medical wards (7, 8, 9 and 16)) and Short Staying Unit (SSU) were selected for rest of the project.

\section{Study population}

There were 26 Medical Laboratory Technologist (MLT) working at the institution including chief MLT. All of them were selected for this project because as a practice they have been rotated within 6 months period between 4 main sections of the lab.

There were 104 Nursing Officers (NOs) and 4 sisters working in the selected wards and SSU. All of them were selected for the project.

Director, Relevant Consultants, Chief MLT and Sisters /in charge NOs were selected for KIIs.

\section{Study instruments}

Qualitative tools - FGDs, KIIs

Quantitative tools - Interviewer administered Questionnaires for MLTs and NOs

Indicators to measure the quality of the laboratory process and results

Percentage of samples with appropriate container

Percentage of samples with appropriate volume

Availability of selected practices in the laboratory process

Usefulness of the QA process to deliver quality results

\section{Study implementation}




\section{Study execution}

$\boldsymbol{Q A}$ committee was established

Training programmes on $Q A$

Error reporting system/Nonconformity handling form was developed

Workshops were conducted

Sample collection counter and facilitator service was introduced

A sample collection manual was prepared

Internal circular was issued

\section{$\underline{\text { Data collection }}$}

This was done by the Principal Investigator (PI) with four trained medical students.

\section{Data analysis}

Data gathered from surveys was analyzed using the Statistical Package for the Social Sciences data analyzing software (SPSS) software

\section{Ethical and Administrative approval}

Ethical approval was obtained from the Ethical Review Committee of Faculty of Medicine, University of Colombo.

Approval for the project proposal was obtained from the Board of Study in Medical Administration. Permission was obtained from Director DGHK.

\section{$\underline{\text { Risks and Benefits }}$}

The study was not a human interventional type and there was no risk to the participants. $\underline{\text { Results }}$

$\underline{\text { Pre intervention }}$

KIIs

Defect in sample collection procedures due to lack of awareness.

Lack of proper methods to transport the samples

Unavailability of visible Standard Operating Procedures

Non availability of error reporting system

Lack of systematic mechanism to record keeping

Unavailability of report sending system

FGDs

Non availability of continuous training and workshops to improve awareness

Non availability of assign worker for responsibility of sending the samples

\section{Post intervention}

KIIs

There has been significant reduction in sample collection errors

There has been significant reduction in transportation errors Standard Operating Procedures displayed near the equipment

Documentation of sending the samples were improved

Report sending delay were significantly reduced

ample errors were recorded

FGDs

Awareness has been improved due to continuous training programmes and workshops

Samples sending process was streamlined

Awareness has been improved due to continuous training programmes and workshops

Samples sending process was streamlined

Testing errors such as sample preparation defects including human errors were corrected using awareness. 
Shortcomings of entering patient's information to the machines were also reduced

\section{Quantitative assessment}

Only few MLTs (8\%) experienced that samples sent were $100 \%$ in an appropriate container before the intervention but $52 \%$ of MLTs experienced that samples sent were in an appropriate container after the intervention. There was a significant improvement $(\mathrm{P}=0.000)$ in perception of MLTs that samples sent were in an appropriate container after the interventions at 5\% significance level.

None of the MLTs experienced that samples sent were $100 \%$ in an appropriate volume before the intervention but $44 \%$ of MLTs experienced that samples sent were in an appropriate volume after the intervention. There was a significant improvement $(\mathrm{P}=0.000)$ in perception of MLTs that samples sent were in an appropriate volume after the interventions at 5\% significance level

Over $95 \%$ of MLTs perceived that QA process is useful to deliver quality results both before and after the intervention. But only $82 \%$ of nurses perceived that QA process is useful to deliver quality results before the intervention and this was increased to 93\% after the intervention. Perception of NOs in this regard has improved significantly $(\mathrm{P}=0.000)$ after the intervention. However Above table shows $\mathrm{p}=0.070(\mathrm{p}>0.05)$ and the relationship was statistically not significant in MLTs response of usefulness of the QA process to deliver quality results. This is because most of MLTs new QA process is useful to deliver quality results.

\section{Conclusions}

Laboratory investigations are of vital importance in clinical diagnosis. This system at DGHK was found to have several gaps which prevented it from supplying accurate results. Gaps identified, during pre-interventional assessment were under 3 phases of laboratory cycle. Specimen collection, specimen quality, and the specimen transport. They were in the pre analytical phase equipment errors and related errors in the procedures in the analytical phase of the cycle. Further results reporting, record keeping, and distribution of reports were in the post analytical phase.

Reduction of process gaps were achieved by designing interventions to improve the QA system using modified Lang's framework model for change adopted through the QA committee.

Post interventional assessment showed that the sample collection manual which was developed to improve pre analytical stage of laboratory cycle had helped in reduction of errors in relation to specimen collection, quality, and the transportation. Related errors in the procedures in the analytical phase were minimized with the improvement of knowledge and the awareness of the MLTs Further, sample collection counter, the record keeping mechanisms and report delivery systems were effective in improving post analytical errors. In addition, when comparing pre and post interventional assessment there was a significant reduction in TAT. Apart from usefulness of the QA process to deliver quality results by MLTs, all the other measured variables by MLTs and NOs were statistically significant. 
When evaluating this project, process and outcome indicators have shown that the strategies implemented for improving laboratory quality assurance process at DGHK were successful.

\section{REFERENCES}

Agarwal, R. 2014. Quality-Improvement Measures as Effective Ways of Preventing Laboratory Errors. Laboratory Medicine 45(2), pp. e80-e88. doi: 10.1309/LMD0YIFPTOWZONAD. Aldosari, B. et al. 2017. Surveying the influence of laboratory information system: An end-user perspective. Informatics in Medicine Unlocked 9, pp. 200-209. doi:

10.1016/j.imu.2017.09.002.

Amukele, T.K. et al. 2012. External Quality Assurance

Performance of Clinical Research Laboratories in Sub-Saharan

Africa. American Journal of Clinical Pathology 138(5), pp. 720723. doi: 10.1309/AJCP8PCM4JVLEEQR.

Angeletti, S. et al. 2015. Laboratory Automation and IntraLaboratory Turnaround Time: Experience at the University Hospital Campus Bio-Medico of Rome. Journal of Laboratory Automation 20(6), pp. 652-658. doi: $10.1177 / 2211068214566458$.

Beyanga, M. et al. 2018. Implementation of the laboratory quality management system (ISO 15189): Experience from Bugando Medical Centre Clinical Laboratory - Mwanza, Tanzania. African Journal of Laboratory Medicine 7(1). Available at:

http://www.ajlmonline.org/index.php/AJLM/article/view/657 [Accessed: 23 February 2020].

Bhaskar, H.L. 2018. Business process reengineering framework and methodology: a critical study. International Journal of Services and Operations Management 29(4), p. 527. doi: 10.1504/IJSOM.2018.090456.

Derua, Y.A. et al. 2011. Users' and health service providers' perception on quality of laboratory malaria diagnosis in Tanzania. Malaria Journal 10(1), p. 78. doi: 10.1186/1475-287510-78.

Diana, N.T.S. 2005. Understanding Clinical Governance and Quality Assurance; Making it happen.

Garfiled, F. 1991. Quality Assurance Principles for Analytical Laboratories. Association of Official Analytical Chemists. Arlington, VA.

Guevara, G. et al. 2014. The impact of SLMTA in improving laboratory quality systems in the Caribbean Region. African Journal of Laboratory Medicine 3(2), p. 9 pages. doi: 10.4102/ajlm.v3i2.199.

Gunatilaka, M. 2012. Quality Assurance of laboratory test results at the Medical Research Institute "We maintain quality and others to follow".
Hammerling, J.A. 2012. A Review of Medical Errors in Laboratory Diagnostics and Where We Are Today: Table 1. Laboratory Medicine 43(2), pp. 41-44. doi: 10.1309/LM6ER9WJR1IHQAUY.

Hawkins, R.C. 2007. Laboratory turnaround time. The Clinical Biochemist. Reviews 28(4), pp. 179-194.

ISO 1994. Quality Management and Quality Assurance - a Vocabulary. International Organization for Standardization, Geneva.

Kaoje, A.U. et al. 2017. Quality of Medical Laboratory Services in a Tertiary Health Institution in Sokoto, Nigeria., pp. 4(4):246259.

Khoury, M. et al. 1996. Error rates in Australian chemical pathology laboratories. The Medical Journal of Australia 165(3), p. p.128.

Koike, S. and Takeda, T. 2017. Experimental Research of Blood Collected From the Peripheral Side of the Fluid Infusion Site That Is Not Affected by Fluid Infusion. Journal of Hematology 6(1), pp. 1-5. doi: 10.14740/jh318e.

Lukić, V. 2017. Laboratory Information System - Where are we Today? Journal of Medical Biochemistry 36(3), pp. 220-224. doi: 10.1515/jomb-2017-0021.

Marques-Garcia, F. et al. 2015. Importance of implementing an analytical quality control system in a core laboratory.

Nevalainen, D. et al. 2000. Evaluating Laboratory Performance on Quality Indicators With the Six Sigma Scale. Archives of Pathology \& Laboratory Medicine., pp. 516-519.

Pai, S. and Frater, J. 2019. Quality management and accreditation in laboratory hematology: Perspectives from India.

Raab, S.S. 2006. Improving Patient Safety Through Quality Assurance. Archives of Pathology \& Laboratory Medicine 130(5), pp. 633-637. doi: 10.1043/15432165(2006)130[633:IPSTQA]2.0.CO;2.

Rahman, T. 2011. Quality Assurance (QA) in Laboratory Testing.

Rana, S.V. 2012. No Preanalytical Errors in Laboratory Testing: A Beneficial Aspect for Patients. Indian Journal of Clinical Biochemistry 27(4), pp. 319-321. doi: 10.1007/s12291-0120271-2.

Schultze, A.E. and Irizarry, A.R. 2017. Recognizing and Reducing Analytical Errors and Sources of Variation in Clinical Pathology Data in Safety Assessment Studies. Toxicologic Pathology 45(2), pp. 281-287. doi: 10.1177/0192623316672945. Selvakumar, R. 2010. Good Laboratory Practices. Indian Journal of Clinical Biochemistry 25(3), pp. 221-224. doi:

10.1007/s12291-010-0077-z.

Shiferaw, M.B. and Yismaw, G. 2019. Magnitude of delayed turnaround time of laboratory results in Amhara Public Health Institute, Bahir Dar, Ethiopia. BMC Health Services Research 19(1), p. 240. doi: 10.1186/s12913-019-4077-2. 
Tadeu, B.T.M. and Geelhoed, D. 2016. "This thing of testing our blood is really very important": a qualitative study of primary care laboratory services in Tete Province, Mozambique.

International Journal for Equity in Health 15(1), p. 133. doi: 10.1186/s12939-016-0418-5.

Woodcock, S. et al. 2010. The Role of Standards and Training in Preparing for Accreditation. American Journal of Clinical Pathology 134(3), pp. 388-392. doi: 10.1309/AJCP03TFPBKEYYNT.

Yao, K. et al. 2010. Improving Quality Management Systems of Laboratories in Developing Countries: An Innovative Training Approach to Accelerate Laboratory Accreditation. American Journal of Clinical Pathology 134(3), pp. 401-409. doi: 10.1309/AJCPNBBL53FWUIQJ. 